Appeared in: Probabilistic Engineering Mechanics, 2013, 33: 26-37.

\title{
Stochastic hydroelastic analysis of pontoon-type very large floating structures considering directional wave spectrum
}

Iason Papaioannou ${ }^{1,2^{*}}$, Ruiping $\mathrm{Gao}^{3}$, Ernst $\operatorname{Rank}^{1}$, Chien Ming Wang ${ }^{3}$

${ }^{1}$ Lehrstuhl für Computation in Engineering, Technische Universität München, 80290 Munich, Germany

${ }^{2}$ Engineering Risk Analysis Group, Technische Universität München, 80290 Munich, Germany

${ }^{3}$ Department of Civil and Environmental Engineering, National University of Singapore, Kent Ridge 119260, Singapore

${ }^{*}$ Corresponding author. Email: iason.papaioannou@tum.de

\begin{abstract}
The hydroelastic response of pontoon-type very large floating structures (VLFS) is obtained by resolving the interaction between the surface waves and the floating elastic body. We carry out the analysis in the frequency domain, assuming that the surface waves can be described by a directional wave spectrum. The response spectra can then be computed by application of stationary random vibration analysis. Applying the modal expansion method, we obtain a discrete representation of the required transfer matrices for a finite number of frequencies, while the influence of the wave direction is obtained by numerical integration of the directional components of the spectrum. Moreover, assuming a Gaussian input, we can apply well known approximations to obtain the distribution of extremes. The method is applied to an example VLFS and the effect of different mean wave angles on the stochastic response is investigated.
\end{abstract}

\section{Keywords}

Hydroelasticity, VLFS, directional spectrum, random vibration, response statistics, distribution of extremes. 


\section{Introduction}

Very large floating structure (VLFS) technology is an attractive approach to reclaim land from sea. These structures can and are already being used for floating bridges, floating piers, floating performance stages, floating fuel storage facilities, floating airports, even habitation, and other purposes [1]. The floating structure can be easily constructed, exploited, and relocated, expanded, or removed. These structures are cost-effective and environmentally friendly floating artificial islands.

Pontoon-type VLFSs are relatively flexible floating structures that behave like giants plates resting on the sea surface. Owing to their flexibility and large dimensions, their response is governed by elastic deformations instead of rigid body motions. The hydroelastic response of VLFS is obtained by resolving the interaction between the surface waves and the floating elastic body. Various methods have been proposed for the hydroelastic analysis of VLFS [2]. One of the most widely used approaches for performing the hydroelastic analysis in the frequency domain is the modal expansion method [3] that utilizes the dry modes of the floating plate. In the open literature, however, the response is usually obtained for distinct wave frequencies and wave angles. In order to obtain a robust VLFS design against waveinduced deformations and stresses, it is necessary to account for the stochastic nature of wind waves. However, there is limited published work on predicting the stochastic response of VLFS. Hamamoto [4] derived analytical expressions for the response of large circular floating structures subject to a spectrum of wave frequencies. Chen et al. [5-7] studied the influence of second-order effects of the structural geometry and wave forces on the response of VLFS under two irregular wave systems coming from different directions.

In this paper, we develop a method for hydroelastic analysis of VLFS subject to a directional wave spectrum. The analysis is carried out in the frequency domain by application of the modal expansion method. The fluid domain is discretized by the boundary element method, while for the structure we use the finite element method derived from the Mindlin plate theory, that allows for the effects of transverse shear deformation and rotary inertia. The derived linear system allows for the application of linear random vibration theory for the evaluation of response spectra. Assuming that the wind wave can be described by a Gaussian process, we can estimate the distribution of extremes and hence obtain mean extreme values of response quantities that are relevant for design. 


\section{Hydroelastic analysis of VLFS}

\subsection{Plate-water model}

Figure 1 shows the schematic diagram of the coupled plate-water problem. The VLFS has a length $L$, width $B$, height $h$ and is assumed to be perfectly flat with free edges. A zero draft is assumed for simplicity. The water is treated as an ideal fluid (inviscid and incompressible) and its flow is irrotational. The water domain is denoted by $\Delta$. The symbols $\Omega_{H B}, \Omega_{F}$ and $\Omega_{S B}$ represent the plate domain, the free water surface boundary and the seabed boundary, respectively. The free and undisturbed water surface is at $z=0$ while the seabed is found at $\mathrm{z}$ $=-H$. Assuming an incident wave $\phi_{I}$ with a circular frequency $\omega$, height $2 A$ and wave angle $\theta$ enters the computational domain, the water motion and plate deflection will oscillate in a steady state harmonic motion in the same frequency $\omega$. The deflection $w$ of the plate is measured from the free and undisturbed water surface.

$\mathbf{a}$

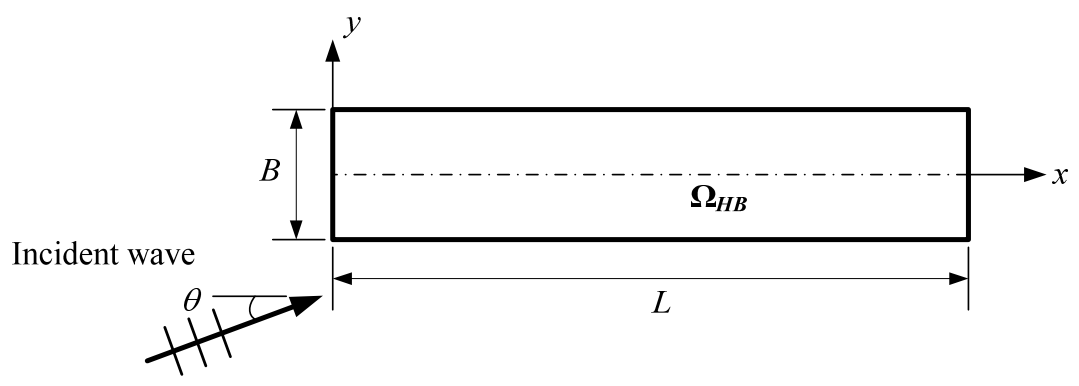

b

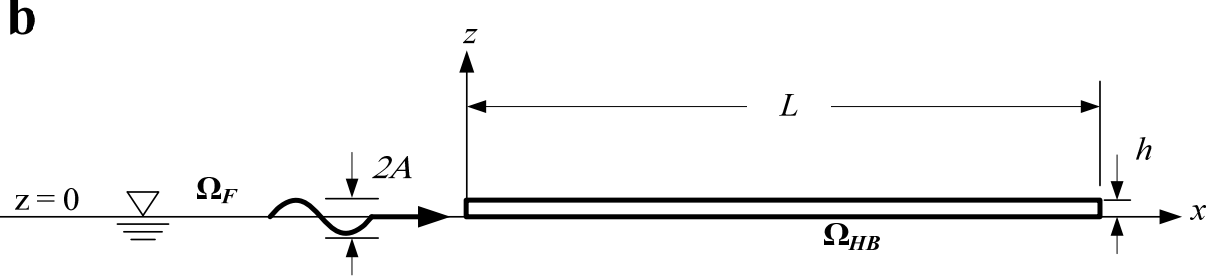

$\begin{array}{lll}z=-H & \mathbf{\Omega}_{S B} & \Delta\end{array}$

$\Delta$

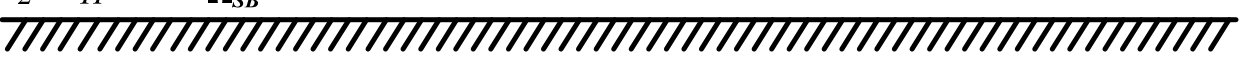

Figure 1. Schematic diagram of coupled plate-water problem (a) plan view (b) side view

\subsection{Equations of motion for floating plate}

The VLFS is modelled as an isotropic and elastic plate based on the Mindlin plate theory [8]. The motion of the Mindlin plate is represented by the vertical displacement $w(x, y)$, the rotation $\psi_{x}(x, y)$ about the $y$-axis and the rotation $\psi_{y}(x, y)$ about the $x$-axis. The governing equations of motion for the Mindlin plate (after omitting the time factor $e^{-i \omega t}$ ) are given by 


$$
\begin{aligned}
& \kappa^{2} G h\left[\left(\frac{\partial^{2} w}{\partial x^{2}}+\frac{\partial^{2} w}{\partial y^{2}}\right)+\left(\frac{\partial \psi_{x}}{\partial x}+\frac{\partial \psi_{y}}{\partial y}\right)\right]+\rho_{p} h \omega^{2} w=p(x, y) \\
& D\left[\frac{(1-v)}{2}\left(\frac{\partial^{2} \psi_{x}}{\partial x^{2}}+\frac{\partial^{2} \psi_{x}}{\partial y^{2}}\right)+\frac{(1+v)}{2}\left(\frac{\partial^{2} \psi_{x}}{\partial x^{2}}+\frac{\partial^{2} \psi_{y}}{\partial x \partial y}\right)\right]-\kappa^{2} G h\left(\frac{\partial w}{\partial x}+\psi_{x}\right)+\rho_{p} \frac{h^{3}}{12} \omega^{2} \psi_{x}=0 \\
& D\left[\frac{(1-v)}{2}\left(\frac{\partial^{2} \psi_{y}}{\partial y^{2}}+\frac{\partial^{2} \psi_{y}}{\partial x^{2}}\right)+\frac{(1+v)}{2}\left(\frac{\partial^{2} \psi_{y}}{\partial y^{2}}+\frac{\partial^{2} \psi_{x}}{\partial x \partial y}\right)\right]-\kappa^{2} G h\left(\frac{\partial w}{\partial y}+\psi_{y}\right)+\rho_{p} \frac{h^{3}}{12} \omega^{2} \psi_{y}=0
\end{aligned}
$$

where $G=E /[2(1+v)]$ is the shear modulus, $\kappa^{2}$ the shear correction factor taken as $5 / 6, \rho_{p}$ the mass density of the plate, $h$ the thickness of the plate, $D=E h^{3} /\left[12\left(1-v^{2}\right)\right]$ the flexural rigidity, $E$ the Young's modulus and $v$ the Poisson ratio. The pressure $p(x, y)$ in Eq. (1) comprises the hydrostatic and hydrodynamic pressure, i.e.

$$
p(x, y)=-\rho g w+i \omega \rho \phi(x, y, 0) \text { on } \Omega_{H B}
$$

where $\rho$ is the mass density of water, $i$ the imaginary number $(i=\sqrt{-1}), g$ the gravitational acceleration and $\phi(x, y, 0)$ the velocity potential of water on undisturbed water surface. At the free edges of the floating plate, the bending moment, twisting moment and transverse shear force must vanish, i.e.

$$
\begin{aligned}
& \text { Bending moment } M_{n n}=D\left[\frac{\partial \psi_{n}}{\partial n}+v \frac{\partial \psi_{s}}{\partial s}\right]=0 \\
& \text { Twisting moment } M_{n s}=D\left(\frac{1-v}{2}\right)\left[\frac{\partial \psi_{n}}{\partial s}+\frac{\partial \psi_{s}}{\partial n}\right]=0 \\
& \text { Shear force } Q_{n}=\kappa^{2} G h\left[\frac{\partial w}{\partial n}+\psi_{n}\right]=0
\end{aligned}
$$

where $s$ and $n$ denote the tangential and normal directions to the cross-section of the plate, respectively. 


\subsection{Equations of motion for water}

The water is assumed to be an ideal fluid and has an irrotational flow so that a velocity potential exists. Thus the single frequency velocity potential of water must satisfy the Laplace's equation [9]

$$
\nabla^{2} \phi(x, y, z)=0 \quad \text { in } \Delta
$$

and the boundary conditions

$$
\begin{array}{ll}
\frac{\partial \phi}{\partial z}(x, y, 0)=-i \omega w(x, y) & \text { on } \Omega_{H B} \\
\frac{\partial \phi}{\partial z}(x, y, 0)=\frac{\omega^{2}}{g} \phi(x, y, 0) & \text { on } \Omega_{F} \\
\frac{\partial \phi}{\partial z}(x, y,-H)=0 & \text { on } \Omega_{S B}
\end{array}
$$

Equation (7) implies that no gap exists between the plate and the water-free surface whereas Eq. (8) is derived from the linearized Bernoulli equation where the pressure is taken as zero at the water surface. Equation (9) is the boundary condition at the seabed which expresses impermeability, i.e. no fluid enters or leaves the seabed and hence the velocity component normal to the seabed is zero.

The wave velocity potential must also satisfy the Sommerfeld radiation condition as $|\mathbf{x}| \rightarrow \infty[10]$

$$
\lim _{|\mathbf{x}| \rightarrow \infty} \sqrt{|\mathbf{x}|}\left(\frac{\partial}{\partial|\mathbf{x}|}-i k\right)\left(\phi-\phi_{I}\right)=0 \text { on } \Omega_{\infty}
$$

where $\mathbf{x}=(x, y, z)$ and $\Omega_{\infty}$ represents the artificial fluid boundary at infinity. The wave number $k$ satisfies the dispersion relationship

$$
k \tanh (k H)=\frac{\omega^{2}}{g}
$$

and $\phi_{I}$ is the incident velocity potential given by 


$$
\phi_{I}=A \frac{g}{\omega} \frac{\cosh (k(z+H))}{\cosh k H} e^{i k(x \cos \theta+y \sin \theta)}
$$

where $A$ is the wave amplitude and $\theta$ is the incident wave angle as shown in Fig. 1.

\subsection{Modal expansion of plate motion and water velocity potential}

Equations (1) and (4) indicate that the response of the plate $w(x, y)$ is coupled with the fluid motions or velocity potential $\phi(x, y, z)$. On the other hand, the fluid motion can only be obtained when the plate deflection $w(x, y)$ is specified in the boundary condition at the fluid side of the fluid-structure interface, as given in Eq. (7). In order to decouple this fluidstructure interaction problem into a hydrodynamic problem in terms of the velocity potential and a plate vibration problem in terms of the generalized displacements, we adopt the modal expansion method as proposed by Newman [3]. According to this method, the deflection of the plate $w(x, y)$ is expanded as a finite series of products of the modal functions $c_{l}^{w}(x, y)$ and corresponding complex amplitudes $\varsigma_{l}$ :

$$
w(x, y)=\sum_{l=1}^{N} \varsigma_{l} c_{l}^{w}(x, y)
$$

where $N$ is the number of terms in the series. The single frequency velocity potential $\phi$ of water can be separated into the diffracted part $\phi_{D}$ and the radiated part $\phi_{R}$ based on the linear potential theory. The radiated potential $\phi_{R}$ can be further decomposed as [11]

$$
\phi(x, y, z)=\phi_{D}(x, y, z)+\phi_{R}(x, y, z)=\phi_{D}(x, y, z)+\sum_{l=1}^{N} \varsigma_{l} \phi_{l}(x, y, z)
$$

where $\phi_{l}(x, y, z)$ is the radiation potential corresponding to the unit-amplitude motion of the $l$-th modal function and $\varsigma_{l}$ is the complex amplitude which is assumed to be the same as those given in Eq. (13) [3]. The diffracted potential $\phi_{D}$ is taken as the sum of the incident potential $\phi_{I}$ and the scattered potential $\phi_{S}$ which represents the outgoing wave from the body. 


\subsection{Hydrodynamic analysis}

By substituting the expanded plate deflection and velocity potential [Eqs. (13) and (14)] into Eq. (6) and the boundary conditions [Eqs. (7) to (10)], we obtain the decoupled Laplace's equation and boundary conditions for each of the uni-amplitude radiation potentials (i.e. for $l$ $=1,2, \ldots, N)$ and the diffraction potential (i.e. for $l=D$ )

$$
\begin{aligned}
& \begin{array}{ll}
\nabla^{2} \phi_{l}(x, y, z)=0 & \text { in } \Delta
\end{array} \\
& \frac{\partial \phi_{l}}{\partial z}(x, y, 0)=\left\{\begin{array}{cl}
-i \omega c_{l}^{w}(x, y) & \text { for } l=1,2, \ldots, N \\
0 & \text { for } l=D
\end{array} \text { on } \Omega_{H B}\right. \\
& \frac{\partial \phi_{l}}{\partial z}(x, y, 0)=\frac{\omega^{2}}{g} \phi_{l}(x, y, 0) \quad \text { on } \Omega_{F} \\
& \frac{\partial \phi_{l}}{\partial z}(x, y,-H)=0 \quad \text { on } \Omega_{S B} \\
& \lim _{|\mathbf{x}| \rightarrow \infty} \sqrt{|\mathbf{x}|}\left(\frac{\partial \phi_{l}}{\partial|\mathbf{x}|}-i k \phi_{l}\right)=0 \quad \begin{array}{l}
\text { for } l=1,2, \ldots, N \quad \text { on } \Omega_{\infty} \\
\text { for } l=S
\end{array}
\end{aligned}
$$

We then transform Eqs. (15) to (19) into an integral equation by using Green's second identity [1]. The resulting boundary integral equation is

$$
\phi_{l}(\mathbf{x})+\int_{\Omega_{\text {Нв }}} \frac{\partial G(\mathbf{x}, \boldsymbol{\xi})}{\partial z} \phi_{l}(\xi) d \xi=\int_{\Omega_{\text {Нв }}} G(\mathbf{x}, \xi) \frac{\partial \phi_{l}(\xi)}{\partial z} d \xi
$$

where $\mathbf{x}=(x, y, z)$ is the source point and $\xi=(\xi, \eta, \zeta)$ the field point. $G(\mathbf{x}, \xi)$ is a freesurface Green's function for water of finite depth that satisfies the seabed boundary condition, water free surface boundary condition and boundary at infinity and is given by [12]

$$
G(\mathbf{x}, \xi)=-\sum_{m=0}^{\infty} \frac{K_{0}\left(k_{m} R\right)}{\pi H\left(1+\frac{\sin 2 k_{m} H}{2 k_{m} H}\right)} \cos k_{m}(z+H) \cos k_{m}(\zeta+H)
$$

where $k_{m}$ is a positive root number satisfying $k_{m} \tanh \left(k_{m} H\right)=-\omega^{2} / g$, with $m \geq 1$ and $k_{0}=i k . K_{0}$ is the modified Bessel function of the second kind and $R$ represents the horizontal distance between $\mathbf{x}$ and $\xi$. 
By further introducing the boundary conditions for $\partial \phi_{l} / \partial z$ into Eq. (20), one obtains

$$
\phi_{l}(\mathbf{x})+\int_{\Omega_{\mathrm{HB}}} \frac{\partial G(\mathbf{x}, \boldsymbol{\xi})}{\partial z} \phi_{l}(\xi) d \xi=\left\{\begin{array}{cl}
\int_{\Omega_{\mathrm{HB}}}-i \omega G(\mathbf{x}, \xi) c_{l}^{w}(\xi) d \xi & \text { for } l=1,2, \ldots, N \\
4 \pi \phi_{I}(\mathbf{x}) & \text { for } l=D
\end{array}\right.
$$

The integral equation given by Eq. (22) can be solved by discretizing the surface of the boundary $\Omega_{H B}$ using the boundary element method. Within this study the constant panel method [13] is applied.

\subsection{Solution for radiated and diffracted potentials}

By rearranging Eq. (22), the radiated potential $\phi_{R}$ and diffracted potential $\phi_{D}$ can be written in matrix forms as

$$
\begin{aligned}
&\left\{\phi_{R}\right\}_{q \times 1}=\left[\tilde{\phi}_{R}\right]_{q \times N}\{\varsigma\}_{N \times 1}=[\tilde{\phi}]_{q \times q}\{\mathbf{w}\}_{q \times 1} \\
&=\left(-i \omega\left[[\mathbf{I}]+\frac{\partial[G]}{\partial z}\right]^{-1}[G]\right)_{q \times q}\left[c^{w}\right]_{q \times N}\{\varsigma\}_{N \times 1} \\
&\left\{\phi_{D}\right\}_{q \times 1}=\left[[\mathbf{I}]+\frac{\partial[G]}{\partial z}\right]_{q \times q}^{-1} 4 \pi\left\{\phi_{I}\right\}_{q \times 1}
\end{aligned}
$$

where $[G]$ is the global matrix for the Green's function, $[\mathbf{I}]$ the identity matrix and $\left[c^{w}\right]$ the matrix containing $N$ eigenvectors (that corresponds to the deflection $w$ of the plate) obtained by performing a free vibration analysis on the Mindlin plate. The subscripts in Eqs. (23a) and (23b) denote the size of the matrix, where $q$ is the total number of degrees of freedom in the plate domain and $N$ the total number of modes.

\subsection{Solution for plate-water linear equation}

The plate equation is solved by using the finite element (FE) method with 8-node Mindlin plate elements that use the substitute shear strain method to avoid shear locking and spurious zero energy modes [14]. By assembling the coupled plate-water Eqs. (1)-(3) into the global form, we obtain 


$$
\left(\left[K_{f}\right]+\left[K_{s}\right]+\left[K_{w}\right]-\omega^{2}[M]-\omega^{2}\left[M_{w}\right]-i \omega\left[C_{w}\right]\right)_{q \times q}\{\mathbf{w}\}_{q \times 1}=\{F\}_{q \times 1}
$$

where $\left[K_{f}\right],\left[K_{s}\right],\left[K_{w}\right],[M],\left[M_{w}\right]$ and $\left[C_{w}\right]$ are the global flexural stiffness matrix, global shear stiffness matrix, global restoring force matrix, global mass matrix, global added mass matrix and global added damping matrix, respectively. As discussed in Section 2.4, the displacement vector $\{\mathbf{w}\}$ may be expanded in an appropriate set of modes $[c]$ as

$$
\{\mathbf{w}\}_{q \times 1}=[c]_{q \times N}\{\varsigma\}_{N \times 1}
$$

Note that $[c]$ can be obtained by performing a free vibration analysis on the Mindlin plate where $[c]=\left[\begin{array}{lll}c^{w} & c^{\psi_{x}} & c^{\psi_{y}}\end{array}\right]^{T} \cdot\left[\begin{array}{c}c^{w}\end{array}\right]$ is the matrix containing $N$ eigenvectors corresponding to the plate deflection, $\left[c^{\psi_{x}}\right]$ and $\left[c^{\psi_{y}}\right]$ are the matrices containing $N$ eigenvectors corresponding to the rotations about $y$ and $x$-axes, respectively. By substituting Eq. (25) into Eq. (24), we obtain

$$
\begin{aligned}
{[c]_{N \times q}^{T}\left(\left[K_{f}\right]+\left[K_{s}\right]+\left[K_{w}\right]-\omega^{2}[M]-\omega^{2}\left[M_{w}\right]-i \omega\left[C_{w}\right]\right)_{q \times q}[c]_{q \times N}\{\varsigma\}_{N \times 1} } & =[c]_{N \times q}^{T}\{F\}_{q \times 1}=\left\{F_{D}\right\}_{N \times 1}
\end{aligned}
$$

where $\left\{F_{D}\right\}=[c]^{T}\{F\}$ is the generalized exciting force. By using the computed velocity potentials [Eqs. (23a) and (23b)], the elements of the global matrices for the added mass $\left[M_{w}\right]$, the added damping $\left[C_{w}\right]$ and exciting force $\left\{F_{D}\right\}$ can be calculated by

$$
\begin{aligned}
& {\left[M_{w}\right]_{q \times q}=-\frac{\rho}{\omega} \operatorname{Im}\left([\tilde{\phi}]_{q \times q}\right)} \\
& {\left[C_{w}\right]_{q \times q}=\rho \operatorname{Re}\left([\tilde{\phi}]_{q \times q}\right)} \\
& \left\{F_{D}\right\}_{N \times 1}=i \omega \rho[c]_{N \times q}^{T}\left\{\phi_{D}\right\}_{q \times 1}
\end{aligned}
$$


Upon solving the coupled plate-water equation (26), we obtain the complex amplitudes $\{\varsigma\}$ and then we back-substitute the amplitudes into Eq. (25) to obtain the deflection and rotations of the plate $\{\mathbf{w}\}$ and hence the stress resultants.

\section{Stochastic formulation}

\subsection{Directional wave spectrum}

Assuming that the irregular (random) wind waves can be described by a zero mean stationary Gaussian process, they can be completely specified by the directional wave spectrum $S(\omega, \theta)$, which represents the distribution of the wave energy in the frequency domain $\omega$ as well as in direction (wave angle) $\theta$. The directional spectrum is generally expressed in terms of the onedimensional frequency spectrum $S(\omega)$ as

$$
S(\omega, \theta)=S(\omega) D(\theta \mid \omega)
$$

where $D(\theta \mid \omega)$ is the directional spreading function and represents the directional distribution of wave energy for a given frequency $\omega$. The conditioning of $D(\theta \mid \omega)$ on $\omega$ implies that the distribution of wave energy in direction (in general) varies with frequency. The function $D(\theta \mid \omega)$ has the following normalization property

$$
\int_{-\pi}^{\pi} D(\theta \mid \omega) d \theta=1
$$

The sea surface $\eta(x, y, t)$ can then be modeled by linear superposition of monochromatic waves of all possible frequencies approaching a point from all possible directions, i.e.

$$
\eta(x, y, t)=\operatorname{Re}\left[\sum_{j} \sum_{l} A_{j l} e^{i\left(k_{j} x \cos \theta_{l}+k_{j} y \sin \theta_{l}-\omega_{j} t+\varepsilon_{j l}\right)}\right]
$$

where $k_{j}, \omega_{j}$ denote the wave number and corresponding frequency of the $j$ th wave component travelling in the direction $\theta_{l}$ and $\varepsilon_{j l}$ are independent random variables uniformly distributed in $[0,2 \pi]$. The amplitudes $A_{j l}$ are obtained from the directional spectrum as 


$$
A_{j l}=\sqrt{2 S(\omega, \theta) \Delta \omega \Delta \theta}
$$

where $\Delta \omega$ and $\Delta \theta$ represent the change in wave frequency and in direction between two consecutive waves. In this study, we use the (one-sided) one-dimensional frequency spectrum proposed by Bretschneider and further developed by Mitsuyasu for the description of fully developed wind waves [15], i.e.

$$
S_{B M}(\omega)=0.257 H_{1 / 3}^{2} T_{1 / 3}^{-4}\left(\frac{\omega}{2 \pi}\right)^{-5} \exp \left[-1.03\left(T_{1 / 3} \frac{\omega}{2 \pi}\right)^{-4}\right]
$$

where $H_{1 / 3}$ is the significant wave height and $T_{1 / 3}$ is the significant wave period. Also, we assume independence of the directional distribution on the wave frequency and adopt the following directional spreading function given by Pierson et al. [16]

$$
D(\theta \mid \omega)=D(\theta)=\left\{\begin{array}{cc}
\frac{2}{\pi}(\cos (\theta-\bar{\theta}))^{2} & \text { for }|\theta-\bar{\theta}| \leq \frac{\pi}{2} \\
0 & \text { for }|\theta-\bar{\theta}|>\frac{\pi}{2}
\end{array}\right.
$$

where $\bar{\theta}$ is the mean wave angle.

\subsection{Stochastic response}

The stochastic hydroelastic response of the VLFS is obtained by applying the linear random vibration theory. Following the approach adopted for the solution for a single frequency and wave angle, we first obtain the elements of the cross-spectral matrix $\left[S_{I I}(\omega)\right]_{q \times q}$ of the vector of incident potentials $\left\{\phi_{I}\right\}_{q \times 1}$ as

$$
\left[S_{I I}(\omega)\right]_{j, l}=\int_{-\pi}^{\pi}\left|H_{I}(\omega)\right|^{2} e^{-i k\left(x_{j} \cos \theta+y_{j} \sin \theta\right)} S(\omega, \theta) d \theta
$$

where $x_{l j}=x_{l}-x_{j}$ and $y_{l j}=y_{l}-y_{j}$ denote the difference of the $x$ and $y$ coordinates of the locations corresponding to the $l$ th and $j$ th degree of freedom, respectively. The function $H_{I}(\omega)$ is the transfer function from the water surface elevation to the incident potential, given by 


$$
H_{I}(\omega)=\frac{g}{\omega} \frac{\cosh (k(z+H))}{\cosh k H}
$$

Furthermore, we obtain the cross-spectral matrix of the force vector as

$$
\left[S_{F F}(\omega)\right]_{q \times q}=\left[H_{F}(\omega)\right]\left[S_{I I}(\omega)\right]\left[H_{F}(\omega)\right]^{*}
$$

where []$^{*}$ denotes the conjugate transpose operator and the complex transfer matrix $\left[H_{F}(\omega)\right]_{q \times q}$ is obtained by combination of Eqs. (23b) and (27c) as

$$
\left[H_{F}(\omega)\right]_{q \times q}=i 4 \pi \omega \rho\left[[\mathbf{I}]+\frac{\partial[G]}{\partial z}\right]_{q \times q}^{-1}
$$

Finally, the cross-spectral matrix of the response is obtained as

$$
\left[S_{w w}(\omega)\right]_{q \times q}=\left[H_{w}(\omega)\right]\left[S_{F F}(\omega)\right]\left[H_{w}(\omega)\right]^{*}
$$

The response transfer matrix $\left[H_{w}(\omega)\right]_{q \times q}$ is given by:

$$
\left[H_{w}(\omega)\right]_{q \times q}=[c]_{q \times N}\left[H_{\varsigma}(\omega)\right]_{N \times N}[c]_{N \times q}^{T}
$$

where $\left[H_{\varsigma}(\omega)\right]_{N \times N}$ is the harmonic transfer matrix, describing the modal response to a harmonic excitation, given by Eq. (26) as

$$
\left[H_{\zeta}(\omega)\right]_{N \times N}=\left([c]^{T}\left(\left[K_{f}\right]+\left[K_{s}\right]+\left[K_{w}\right]-\omega^{2}[M]-\omega^{2}\left[M_{w}\right]-i \omega\left[C_{w}\right]\right)[c]\right)_{N \times N}^{-1}
$$

It should be noted that the inversion in Eq. (40) is trivial (i.e. the matrix to be inverted is diagonal), as the matrix $[c]$ contains the uncoupled modes of the system. 
A discrete representation of the matrix $\left[S_{w w}(\omega)\right]$ is obtained using a finite number of frequencies. We can then compute the $j$ th component of the vector of the $m$ th spectral moment of the response $\left\{\lambda_{w m}\right\}_{q \times 1}$ in terms of the diagonal entries $\left[S_{w w}(\omega)\right]_{j, j}$ of $\left[S_{w w}(\omega)\right]$, as follows:

$$
\left\{\lambda_{w m}\right\}_{j}=\int_{0}^{\infty} \omega^{m}\left[S_{w w}(\omega)\right]_{j, j} d \omega
$$

wherein the integration is performed numerically. The variance of the response can then be obtained by setting $m=0$.

\subsection{Response of stress resultants}

Stress resultants are important in the practical design of VLFS. The stress resultants within a single Mindlin plate element of the floating plate can be calculated by expanding the displacement in the stress resultant-displacement relations using the obtained nodal displacements. The bending and twisting moments within the element $e$ can be obtained as

$$
\left[\begin{array}{l}
M_{x x} \\
M_{y y} \\
M_{x y}
\end{array}\right]=D\left[\begin{array}{ccc}
1 & v & 0 \\
v & 1 & 0 \\
0 & 0 & \frac{(1-v)}{2}
\end{array}\right]\left[B_{f}\right]^{(e)}\{\mathbf{w}\}^{(e)}
$$

where $M_{x x}, M_{y y}$ and $M_{x y}$ are the bending moments and twisting moment per unit length of the plate. The shear forces within the element $e$ can be obtained as

$$
\left[\begin{array}{l}
Q_{x} \\
Q_{y}
\end{array}\right]=\kappa^{2} G h\left[\begin{array}{ll}
1 & 0 \\
0 & 1
\end{array}\right]\left[B_{s}\right]^{(e)}\{\mathbf{w}\}^{(e)}
$$

where $Q_{x}, Q_{y}$ are the transverse shear forces per unit length of the plate. The elemental flexural strain-displacement matrix $\left[B_{f}\right]^{(e)}$ is given by

$$
\left[B_{f}\right]_{3 \times 24}^{(e)}=\left[\begin{array}{llll}
B_{f 1} & B_{f 2} & \cdots & B_{f 8}
\end{array}\right]
$$


where

$$
\left[B_{f i}\right]=\left[\begin{array}{ccc}
0 & \frac{d N_{i}}{d x} & 0 \\
0 & 0 & \frac{d N_{i}}{d y} \\
0 & \frac{d N_{i}}{d y} & \frac{d N_{i}}{d x}
\end{array}\right]
$$

and the shear strain-displacement matrix $\left[B_{s}\right]^{(e)}$ is given by

$$
\left[B_{s}\right]_{2 \times 24}^{(e)}=\left[\begin{array}{llll}
B_{s 1} & B_{s 2} & \cdots & B_{s 8}
\end{array}\right]
$$

where

$$
\left[B_{s i}\right]=\left[\begin{array}{lll}
\frac{d N_{i}}{d x} & N_{i} & 0 \\
\frac{d N_{i}}{d y} & 0 & N_{i}
\end{array}\right]
$$

where $N_{i}(i=1,2, \cdots 8)$ are the basis functions of the 8 -node serendipity Mindlin plate element. Thus, the elemental transfer matrices for moments and shear forces can be expressed as

$$
\begin{aligned}
& \left\{\begin{array}{l}
{\left[H_{M_{x x}}(\omega)\right]^{(e)}} \\
{\left[H_{M_{y y}}(\omega)\right]^{(e)}} \\
{\left[H_{M_{x y}}(\omega)\right]^{(e)}}
\end{array}\right\}=D\left[\begin{array}{ccc}
1 & v & 0 \\
v & 1 & 0 \\
0 & 0 & \frac{(1-v)}{2}
\end{array}\right]\left[B_{f}\right]^{(e)}
\end{aligned}
$$

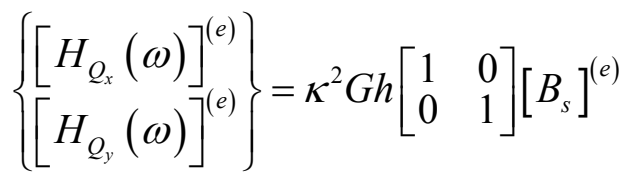

By assembling the elemental transfer matrices, the global transfer matrices for moments and shear forces can then be obtained. Denoting the global transfer matrices of the moments and 
shear forces by $\left[H_{M}(\omega)\right]$ and $\left[H_{Q}(\omega)\right]$, respectively, we can compute the cross-spectral matrices of the stress resultants as follows:

$$
\begin{aligned}
& {\left[S_{M M}(\omega)\right]=\left[H_{M}(\omega)\right]\left[S_{w w}(\omega)\right]\left[H_{M}(\omega)\right]^{*}} \\
& {\left[S_{Q Q}(\omega)\right]=\left[H_{Q}(\omega)\right]\left[S_{w w}(\omega)\right]\left[H_{Q}(\omega)\right]^{*}}
\end{aligned}
$$

The spectral moments of the stress resultants can then be obtained in a manner analogous to Eq. (41).

\subsection{Extreme value prediction}

In the design of VLFS, the knowledge of the distribution of maxima of response quantities over a certain time period is required in order to assess the serviceability and safety of the structure. The distribution of the maxima of any response quantity can be derived using its spectral moments, calculated by Eq. (41). Let $Y(t)$ be the zero mean stationary Gaussian process that describes a response quantity at a certain node and let $\lambda_{m}$ be its $m$-th spectral moment. The distribution $F_{\hat{Y}(T)}(y)$ of $\hat{Y}(T)=\max (Y(t), 0 \leq t \leq T)$, where $T$ is the period of interest, can be expressed as follows [17]:

$$
F_{\hat{Y}(T)}(y)=\exp \left(-\eta_{Y}^{+}(y) T\right)
$$

where $\eta_{Y}^{+}(y)$ is the conditional rate of upcrossings of the level $y$ given the event of no prior upcrossings. Also we should note that Eq. (48) neglects the probability of initial upcrossing of $y$. Assuming that the upcrossings of high levels are independent events, we can approximate $\eta_{Y}^{+}(y)$ with the unconditional upcrossing rate $v_{Y}^{+}(y)$ given by:

$$
v_{Y}^{+}(y)=v_{Y}^{+} \exp \left(-\frac{y^{2}}{2 \sigma_{Y}^{2}}\right)
$$

where $\sigma_{Y}=\sqrt{\lambda_{0}}$ is the standard deviation of $Y$ and $v_{Y}^{+}$is the mean zero upcrossing rate given by: 


$$
v_{Y}^{+}=\frac{1}{2 \pi} \sqrt{\frac{\lambda_{2}}{\lambda_{0}}}
$$

The derived approximation is the well-known Poisson model which is shown to be asymptotically exact for large $T$. However, convergence to the Poisson model becomes slow for narrow-band processes. Vanmarcke [18] gives a more accurate model that accounts for the influence of the bandwidth. According to this model, the conditional upcrossing rate is approximated as follows:

$$
\eta_{Y}^{+}(y) \approx v_{Y}^{+}(y) p(y)
$$

where

$$
p(y)=\left(1-\exp \left[-\left(1-\alpha_{1}^{2}\right)^{0.6} \sqrt{2 \pi} \frac{y}{\sigma_{Y}}\right]\right)\left(1-\exp \left[\frac{-y^{2}}{2 \sigma_{Y}^{2}}\right]\right)^{-1}
$$

wherein $\alpha_{1}=\lambda_{1}\left(\lambda_{0} \lambda_{2}\right)^{-1 / 2}$ is the bandwidth parameter which tends to unity for a narrow-band process.

In code-based design applications, partial safety factors are applied to characteristic values of design quantities, such as displacements and bending moments and designs are checked against serviceability and safety limit-state requirements. Characteristic values are usually taken as the expected maxima of the design quantities over a time period that represents the duration of an extreme event. Accounting for the fact that $\hat{Y}(T)$ follows asymptotically a type I extreme value distribution, we can approximate the expected maximum using the following expression [19]

$$
\mathrm{E}[\hat{Y}(T)] \approx y_{0}+\frac{\gamma}{y_{0}} \sigma_{Y}^{2}
$$

where $\gamma=0.577 \ldots$ is Euler's constant and $y_{0}$ satisfies the following equation:

$$
\frac{y_{0}^{2}}{2 \sigma_{Y}^{2}}=\ln \left[v_{Y}^{+} T\right]+\ln \left[p\left(y_{0}\right)\right]
$$

Equation (54) can be solved for $y_{0}$ by applying an iterative procedure. 


\section{Numerical example}

The VLFS considered by Sim and Choi [20] is used as an example for this study. The length, width and height of the floating plate are 300,60 and $2 \mathrm{~m}$, respectively. The following material properties of the plate are assumed: Poisson's ratio $v=0.13$, Young's modulus $E=$ $1.19 \times 10^{10} \mathrm{~N} / \mathrm{m}^{2}$, and the mass density of the plate $\rho_{p}=256.25 \mathrm{~kg} / \mathrm{m}^{3}$. The water density is $\rho$ $=1025 \mathrm{~kg} / \mathrm{m}^{3}$ and a water depth $H=58.5 \mathrm{~m}$. The finite element mesh of the plate, consisting of 2000 8-node Mindlin elements, is shown in Figure 2. A total number $N=30$ of modes is chosen for the present study. The finite element mesh was chosen fine enough, from our experience with deterministic calculations, so that an accurate solution is obtained for each of the considered wave frequencies for the discrete representation of the transfer matrices.

The chosen parameters for the spectrum of Eq. (32) are $H_{1 / 3}=2 \mathrm{~m}, T_{1 / 3}=6.3 \mathrm{sec}$. In the numerical examples, four cases of mean wave angle $\bar{\theta}$, namely (a) $0^{\circ}$, (b) $30^{\circ}$, (c) $60^{\circ}$ and (d) $90^{\circ}$, are considered. In Figure 3, a plot of the utilized directional wave spectrum for $\bar{\theta}=0^{\circ}$ is shown. The four corner points (P1 to P4, as shown in Fig. 2) and the center point (P5, as shown in Fig. 2) are chosen to illustrate the stochastic behavior of the floating plate.

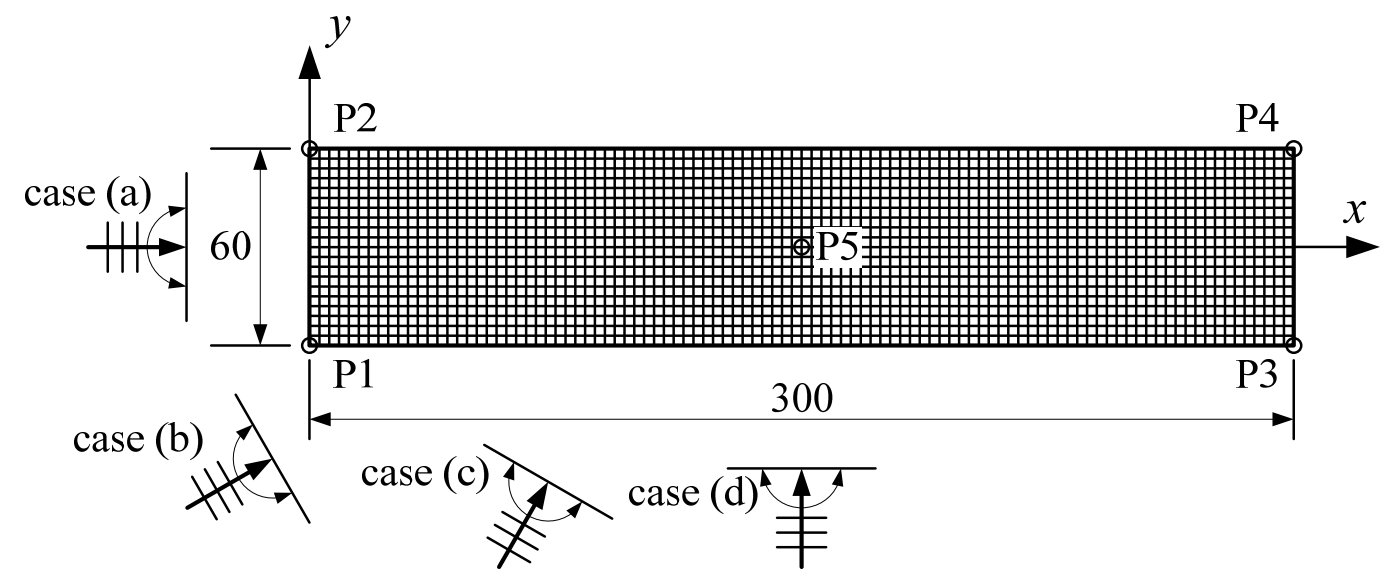

Figure 2. Mesh of the plate 


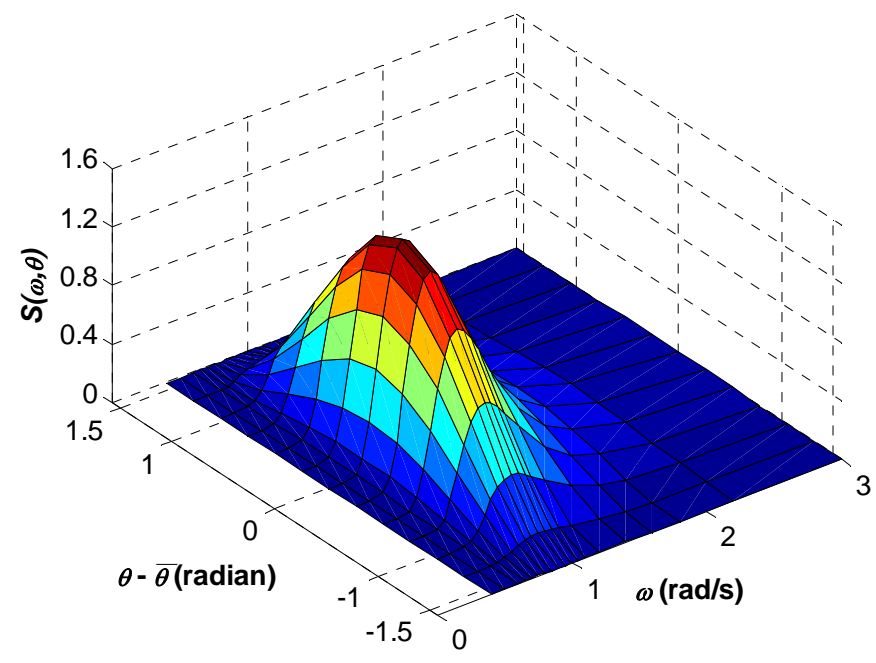

Figure 3. Plot of the applied directional wave spectrum for $\bar{\theta}=0^{\circ}$

\subsection{Stochastic response}

The response spectra of the vertical displacements at the five selected points (P1 to P5, as show in Fig. 2) of the floating structure are obtained as shown in Fig. 4. Figure 5 shows the standard deviation of the vertical displacement for the four mean wave angle cases considered. It can be seen that in general, the overall response of the floating plate increases as the mean wave angle increases from 0 to 90 degrees. In the four cases studied, larger responses are observed in the corner points than the center points. Due to the symmetry of the directional spreading function, we obtain symmetric response spectra for mean wave angle case (a) with respect to the $x$-axis and case (d) with respect to the $y$-axis, respectively, as the effects of oblique wave angles are balanced, as shown in Figs. 4(a) and 4(d). The same results are observed in the plot of the standard deviation of the response, as shown in Figs. 5(a) and 5(d). Owing to this cancellation effect, the response results for these two mean wave angle cases are similar to those obtained by deterministic hydroelastic analysis using these two mean wave angles as distinct wave angles (see results in the Ref. [21] by Gao et al.). 
case (a) $\bar{\theta}=0^{\circ}$

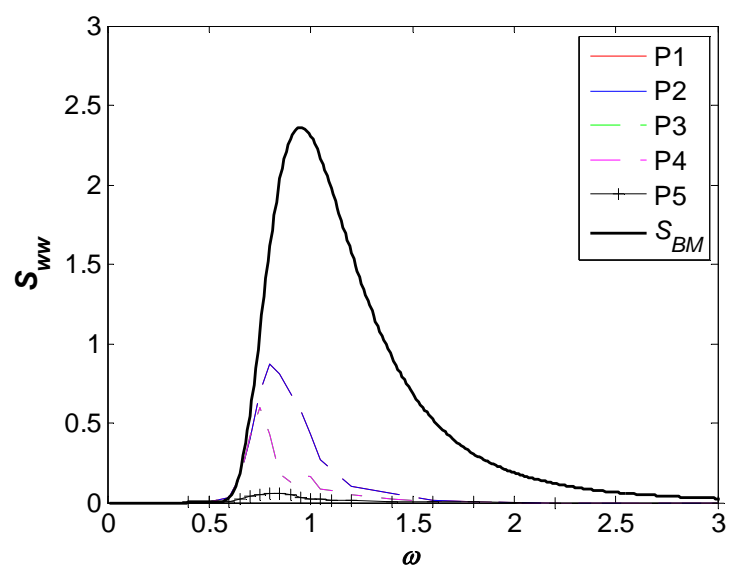

case (c) $\bar{\theta}=60^{\circ}$

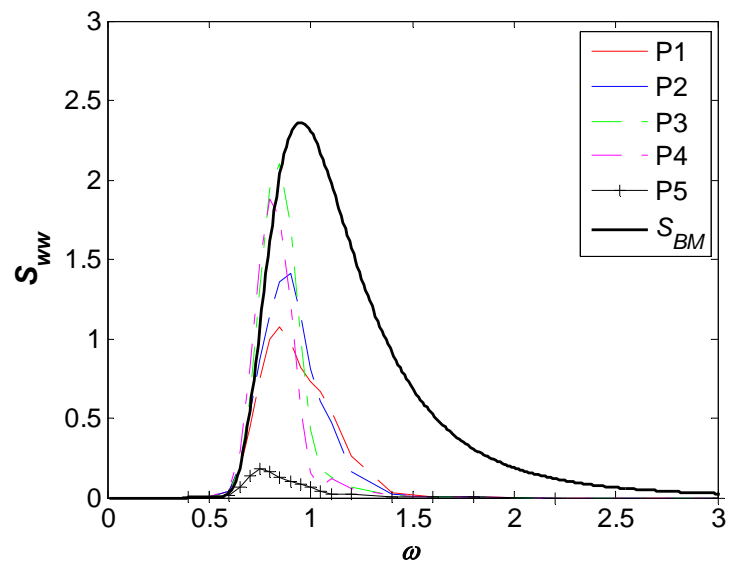

case (b) $\bar{\theta}=30^{\circ}$

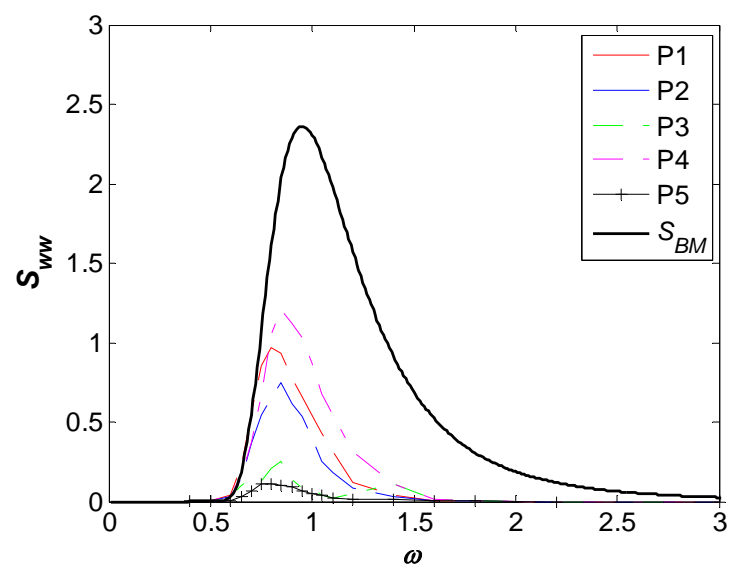

case (d) $\bar{\theta}=90^{\circ}$

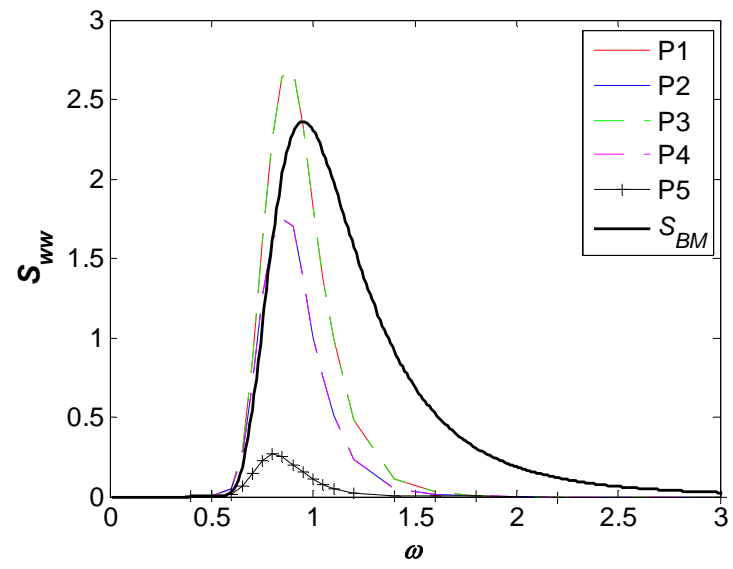

Figure 4. Input spectrum $S_{B M}$ and response spectra of the vertical displacements at 5 selected points for different mean wave angles

However, for other mean wave angle cases, the effects of oblique wave angles cannot be balanced and hence result in different hydroelastic response for the same floating plate. Considering the mean wave angle case (b) for example, the response spectra as shown in Fig. 4(b) is not symmetric, while the largest response is obtained at the corner point P4. This is due to the fact that in this case the directional spectrum includes a larger number of waves coming from other directions which trigger the twisting vibration modes of the plate. This effect can only be captured if a directional spectrum is considered. For the example case with mean wave angle of $30^{\circ}$, neglecting the probability of occurrence of larger oblique wave angles would lead to significantly smaller variances. The same conclusion applies to the mean wave angle case (c), as shown in Fig. 4(c) and Fig. 5(c). 
case (a) $\bar{\theta}=0^{\circ}$

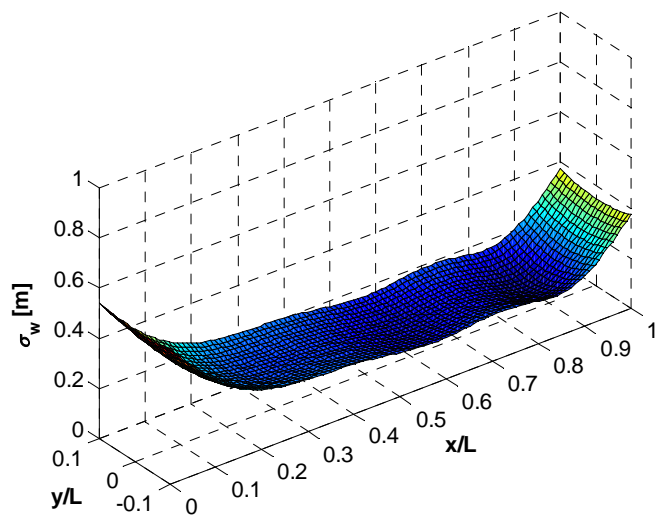

case (c) $\bar{\theta}=60^{\circ}$

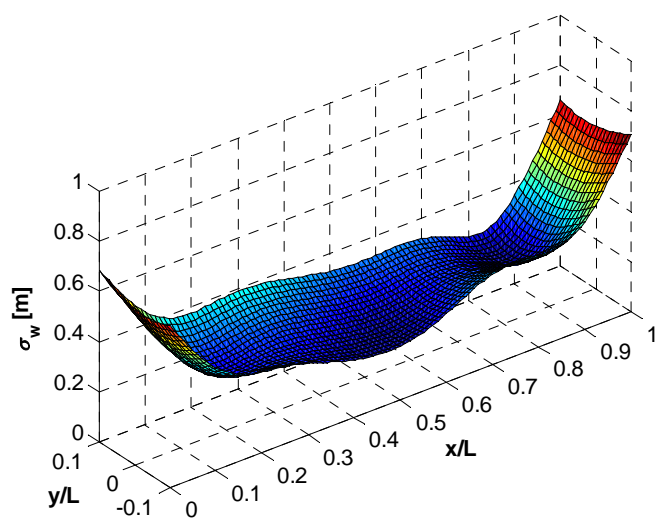

case (b) $\bar{\theta}=30^{\circ}$

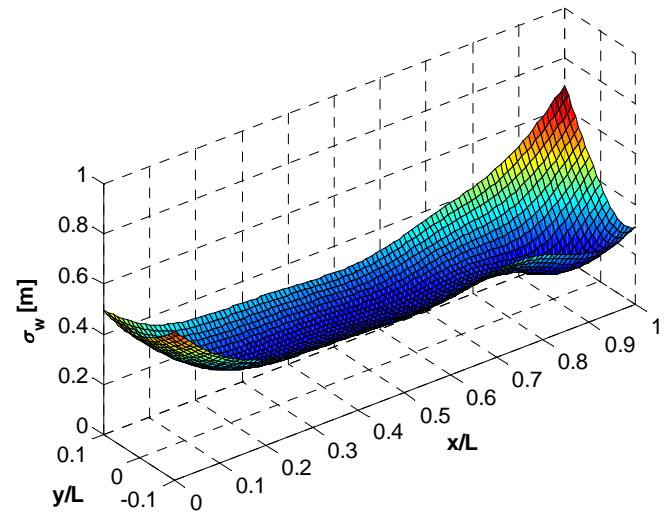

case (d) $\bar{\theta}=90^{\circ}$

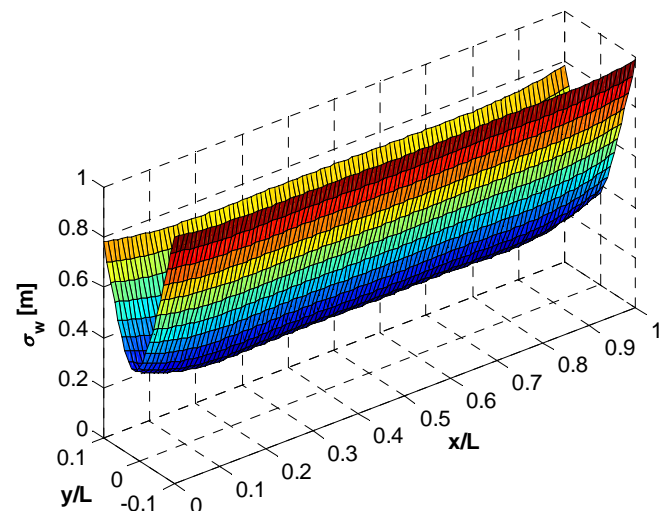

Figure 5. Standard deviations of the vertical displacements for different mean wave angles

\subsection{Response of stress resultants}

In Fig. 6 to Fig. 10, the standard deviations of the stress resultants $M_{x x}, M_{y y}, M_{x y}, Q_{x}$ and $Q_{y}$ are plotted.

Similar to the stochastic response of the displacements, symmetric responses are obtained for the stress resultants $M_{x x}, M_{y y}, M_{x y}, Q_{x}$ and $Q_{y}$ in the cases of mean wave angle $\bar{\theta}=0^{\circ}$ and $\bar{\theta}=90^{\circ}$ as shown in Figs. 6 to 10 . The effect of directional wave is significant in the case of mean wave angle $\bar{\theta}=60^{\circ}$ as shown in Figs. 6(c) and 10(c), where larger standard deviations are obtained as compared to those for $\bar{\theta}=0^{\circ}$. In deterministic analysis, however, smaller values of $M_{x x}$ and $Q_{x}$ are expected in the case of oblique waves because the dominating component ( $x$-direction component) of the wave that results larger value of $M_{x x}$ and $Q_{x}$ is compensated by the $y$-direction component. 
The standard deviations of the moments $M_{y y}$ and $M_{x y}$ are smaller than those of the bending moment $M_{x x}$, as shown in Figs. 6 to 8 . This is due to the large aspect ratio of the floating plate which indicates that the hydroelastic response is significant when the waves are coming along the strong axis of the plate, i.e. the $x$-axis. In the case of mean wave angle $\bar{\theta}=90^{\circ}$, the motion of the plate is dominated by rigid body motion, as can been seen in Fig. 5(d) and Fig. 7(d). However, it is still necessary to investigate the results of twisting moments $M_{x y}$ in the case of oblique mean waves. As shown in Fig. 8(b) and 8(c), the magnitude of the standard deviation of twisting moments $M_{x y}$ are in the same order as those of bending moments $M_{x x}$ results shown in Fig. 6. Moreover, the results of twisting moments $M_{x y}$ are larger for mean wave angle cases (b) and (c) than those for mean wave angle cases (a) and (d).

case (a) $\bar{\theta}=0^{\circ}$

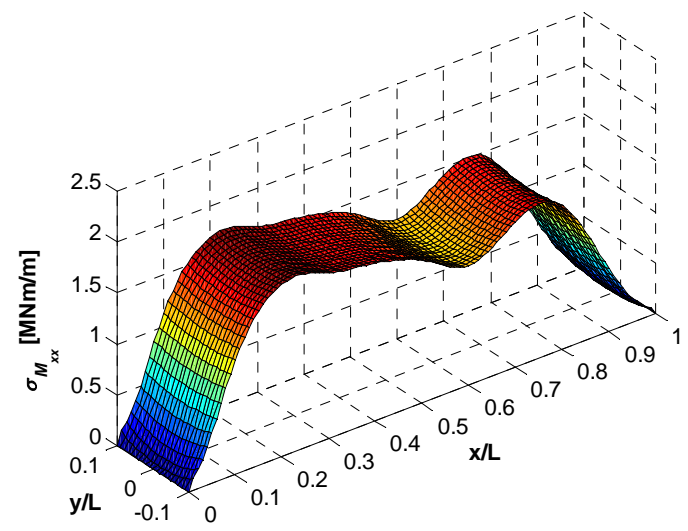

case (c) $\bar{\theta}=60^{\circ}$

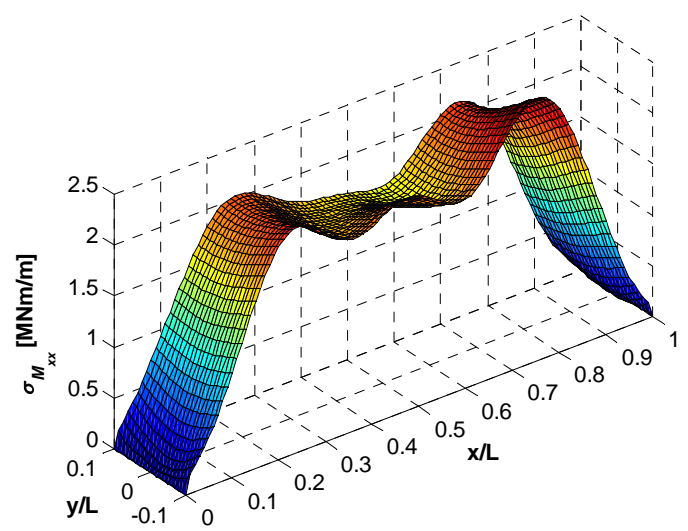

case (b) $\bar{\theta}=30^{\circ}$

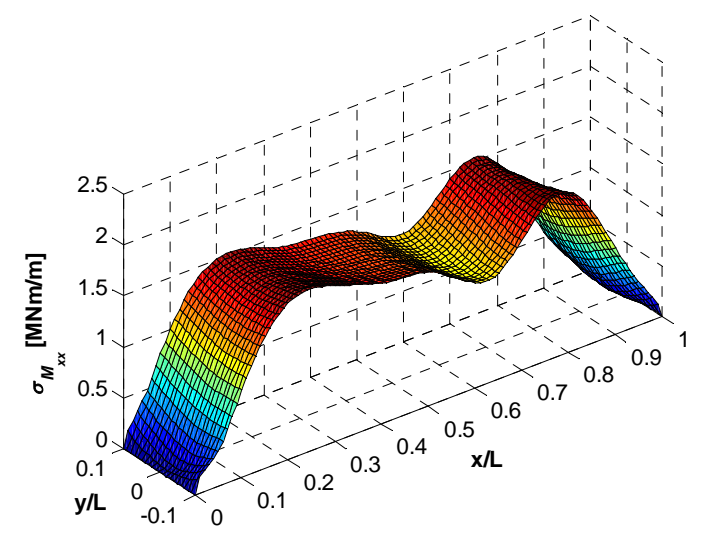

case (d) $\bar{\theta}=90^{\circ}$

Figure 6. Standard deviation of $M_{x x}$ for different mean wave angles 
The blowups at the corners of the plate for the shear forces as shown in Fig. 9 and Fig. 10 are due to the strong variations nature of shear forces near the free edges. As has been discussed by Ramesh et al. [22], the shear forces do not vanish at the free edges, especially at the free corner. However, this effect would not affect the overall observations of the spectra results for shear forces, because it only affects the small portion of the entire domain. Moreover, the exact results of shear forces at these affected regions are known to be vanished.

case (a) $\bar{\theta}=0^{\circ}$

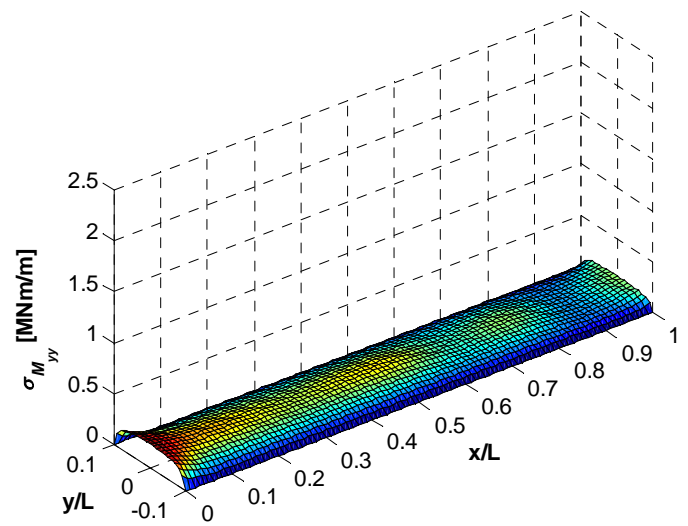

case (c) $\bar{\theta}=60^{\circ}$

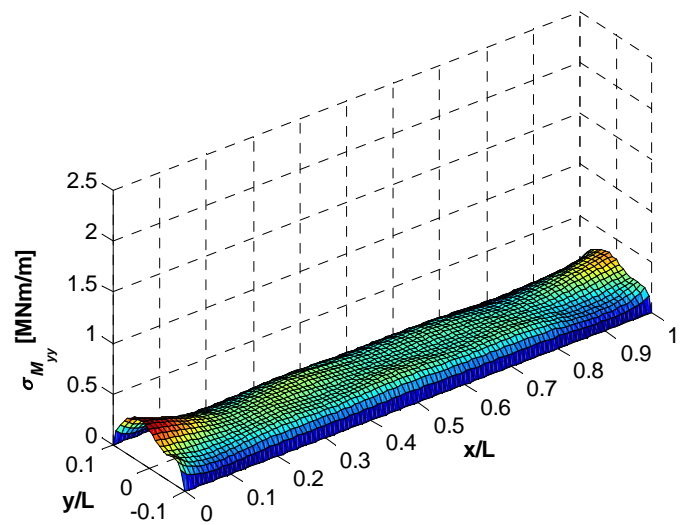

case (b) $\bar{\theta}=30^{\circ}$

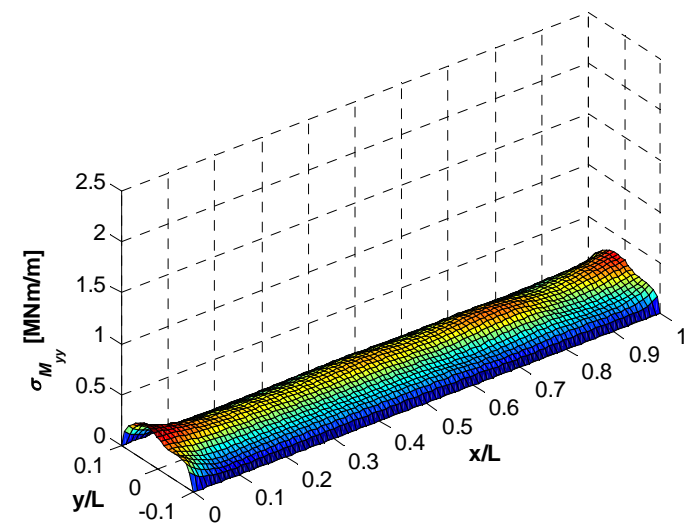

case (d) $\bar{\theta}=90^{\circ}$

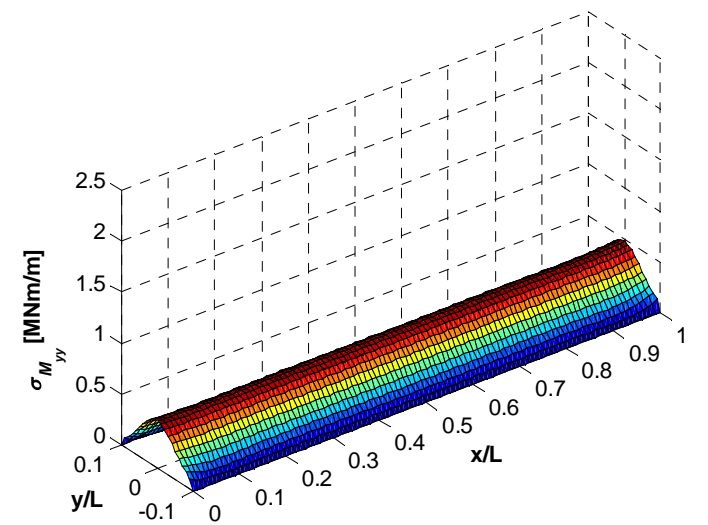

Figure 7. Standard deviation of $M_{y y}$ for different mean wave angles 
case (a) $\bar{\theta}=0^{\circ}$

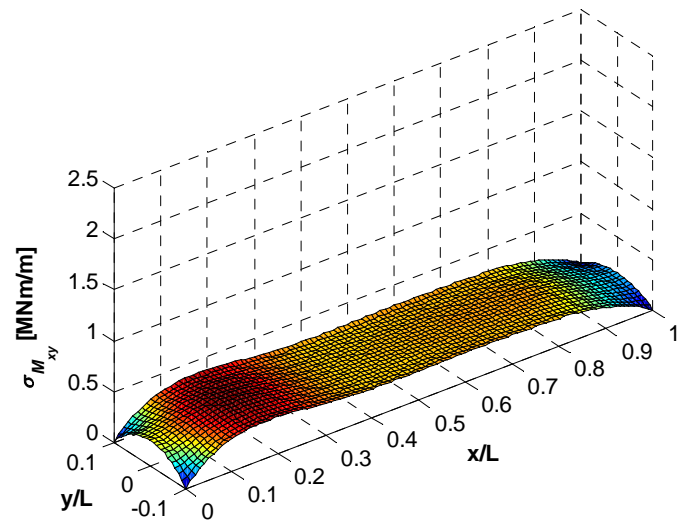

case (c) $\bar{\theta}=60^{\circ}$

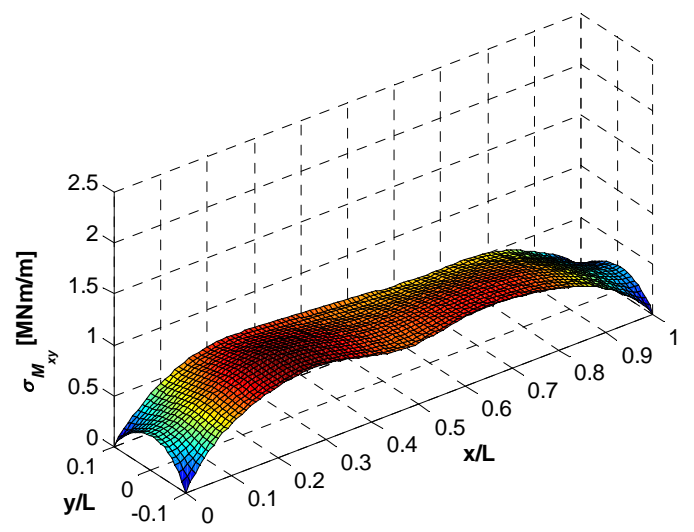

case (b) $\bar{\theta}=30^{\circ}$

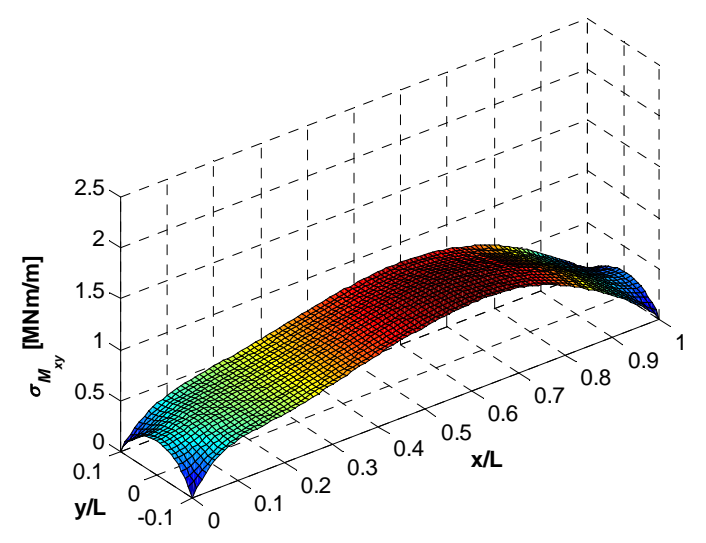

case (d) $\bar{\theta}=90^{\circ}$

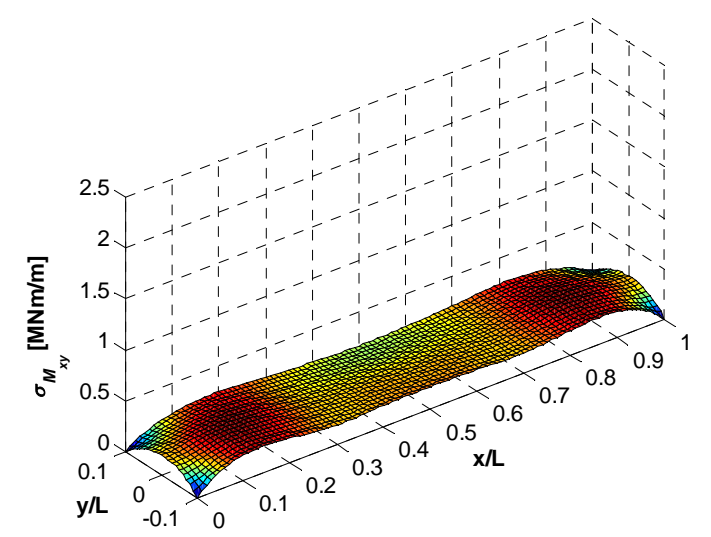

Figure 8. Standard deviation of $M_{x y}$ for different mean wave angles 
case (a) $\bar{\theta}=0^{\circ}$

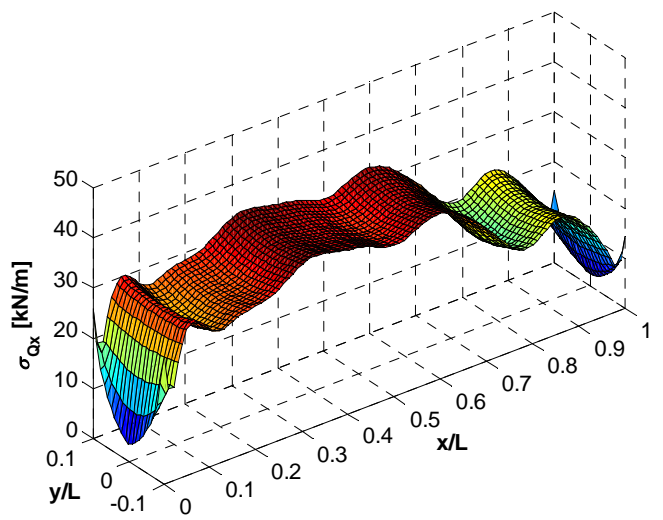

case (c) $\bar{\theta}=60^{\circ}$

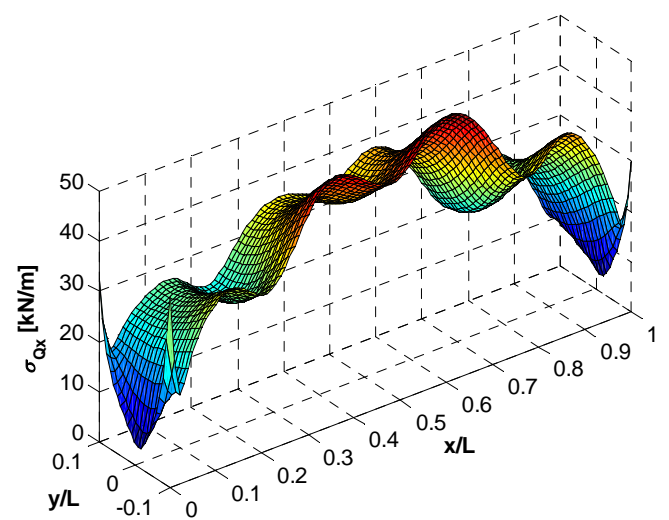

case (b) $\bar{\theta}=30^{\circ}$

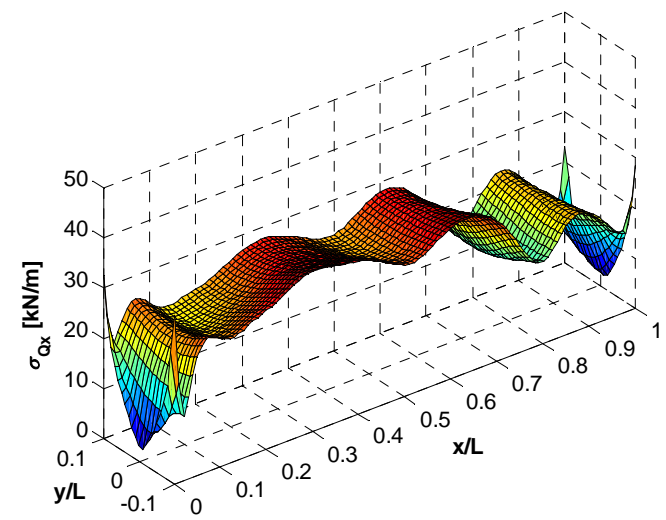

case (d) $\bar{\theta}=90^{\circ}$

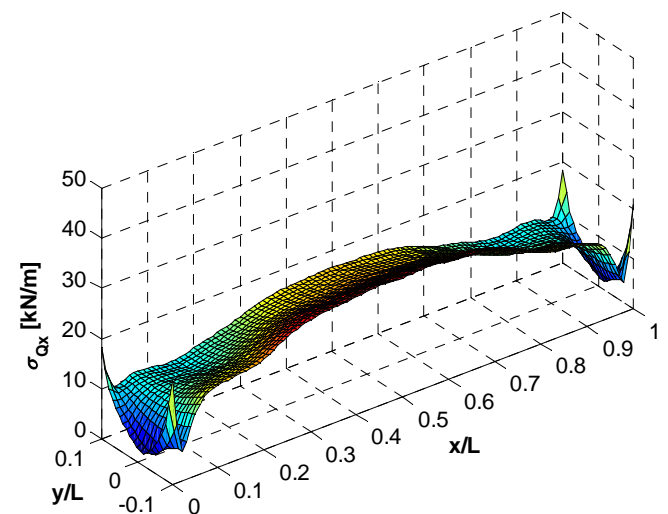

Figure 9. Standard deviation of $Q_{x}$ for different mean wave angles 
case (a) $\bar{\theta}=0^{\circ}$

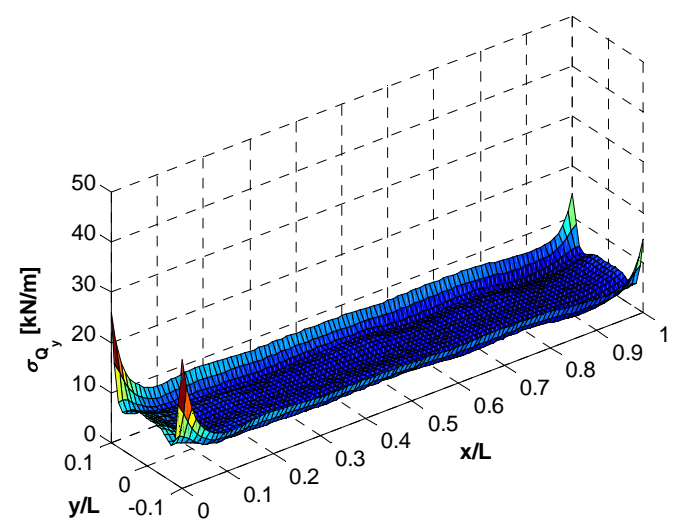

case (c) $\bar{\theta}=60^{\circ}$

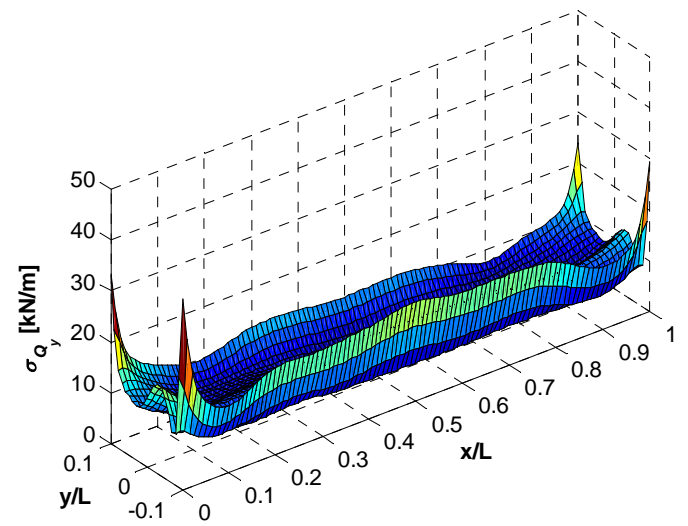

case (b) $\bar{\theta}=30^{\circ}$

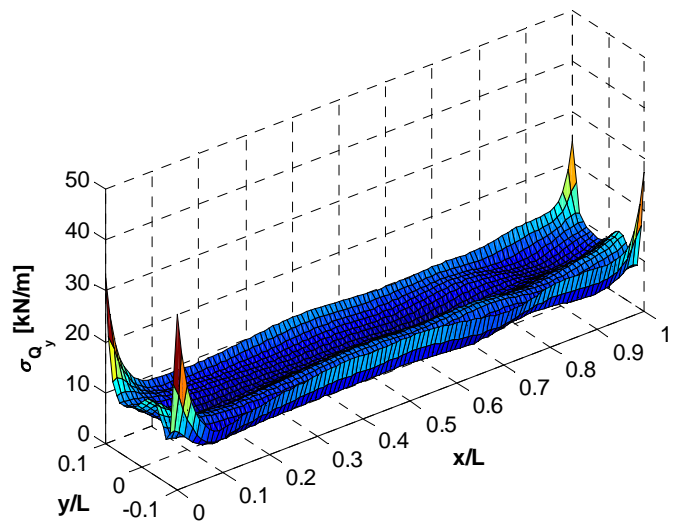

case (d) $\bar{\theta}=90^{\circ}$

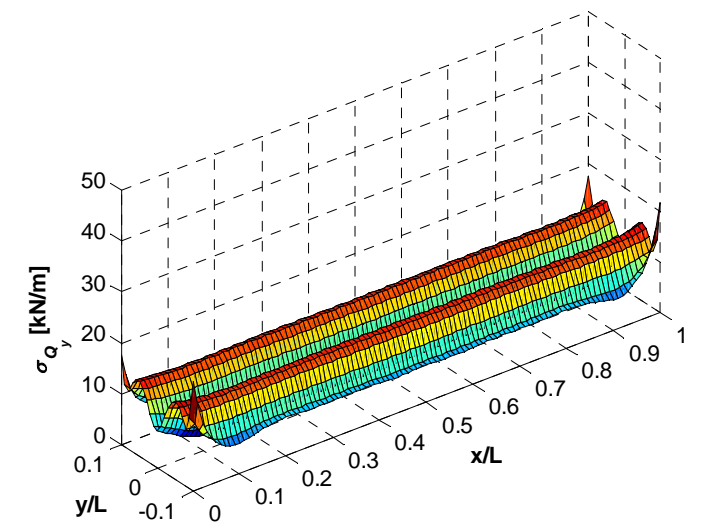

Figure 10. Standard deviation of $Q_{y}$ for different mean wave angles

\subsection{Extreme value prediction}

The expected maxima of the response quantities are obtained based on the Vanmarcke approximation by applying Eq. (53). The period $T$ of interest is set to 2 hours. Figure 11 shows examples of expected maxima of response quantities in the case of mean wave angle $\bar{\theta}=0^{\circ}$.

The maximum value of the expected maximum of stress resultants is extracted for each case of mean wave angles. The results are plotted against the mean wave angle as shown in Fig. 12. It can be seen that bending moment $M_{x x}$ and shear force $Q_{x}$ are the two dominating components of the stress resultants. Large values of these two components are obtained in the cases of mean wave angle $\bar{\theta}=60^{\circ}$ and $\bar{\theta}=75^{\circ}$. This implies that the worst scenario of the floating structure might be in the cases of these mean wave angles and this should be taken into account in the practical design. 

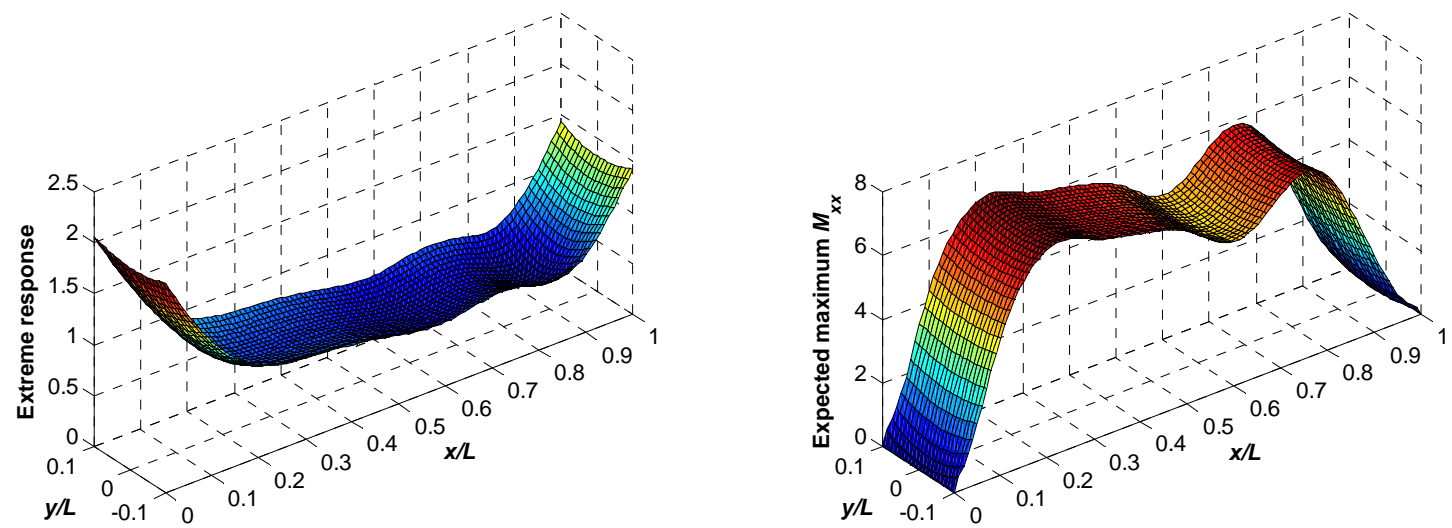

c $M_{y y}$

d $M_{x y}$
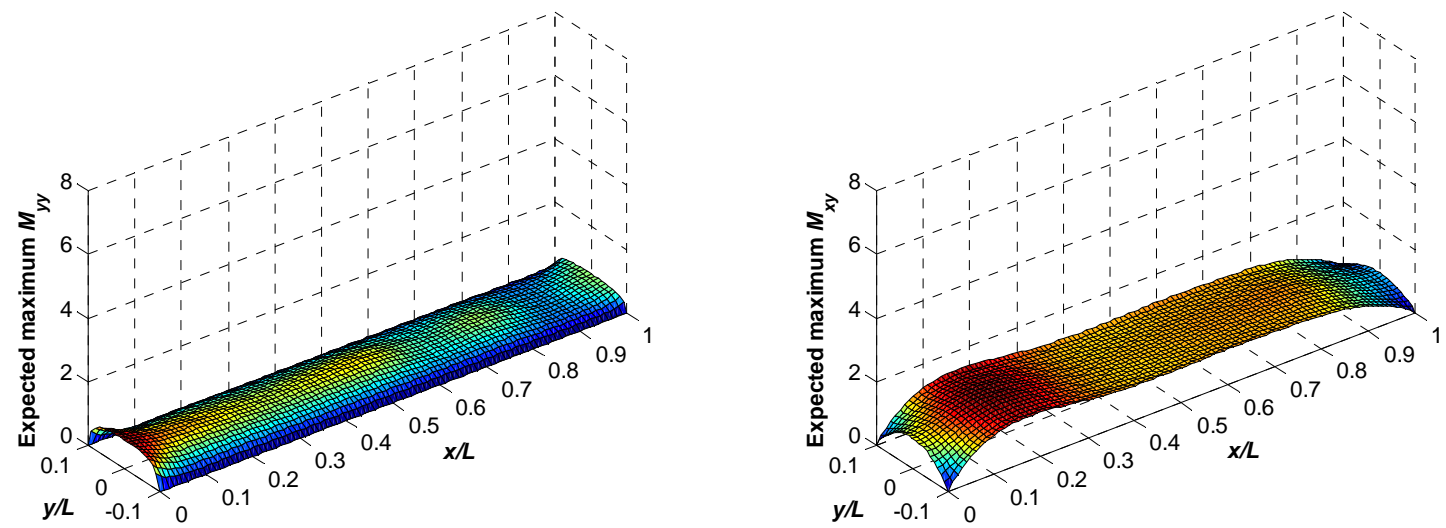

Figure 11. Expected maximum of response quantities predicted by the Vanmarcke approximation for a 2 hours period for $\bar{\theta}=0^{\circ}$.
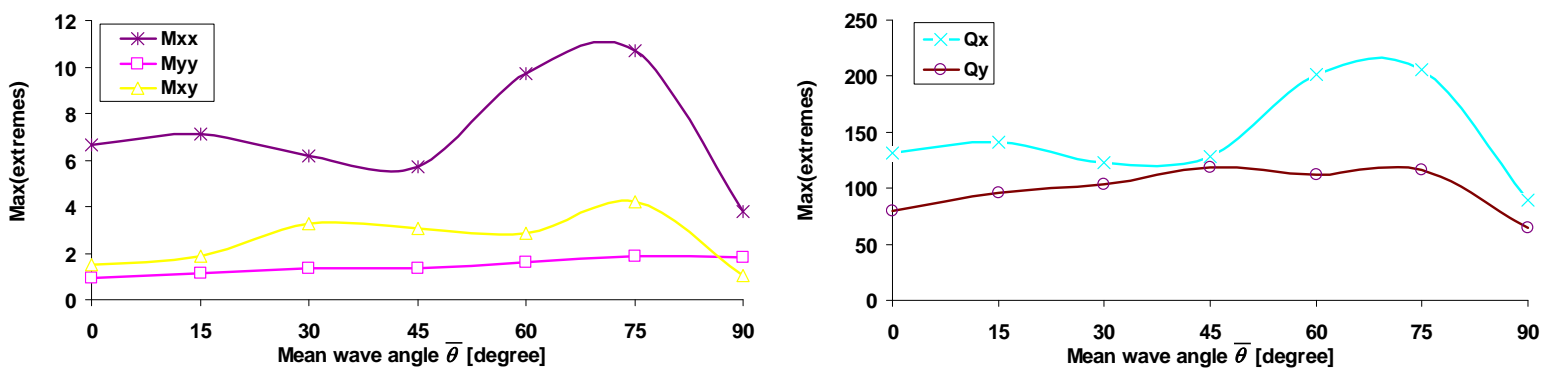

Figure 12. Maximum value of extremes of stress resultants in terms of the mean wave angle 


\section{Concluding remarks}

Based on the linear random vibration theory, a framework for stochastic hydroelastic analysis of very large floating structures subjected to multidirectional irregular waves defined through a directional wave spectrum has been developed. The approach involves a discrete evaluation of the relevant transfer matrices through a numerical resolution of the fluid-structure interaction problem that combines the boundary element method for the fluid potential and the finite element method based on the Mindlin plate theory for the plate response. Spectra of response quantities are obtained as well as extreme responses, assuming a Gaussian input.

The proposed method is applied to the stochastic analysis of a numerical example and the influence of the mean wave angle on the standard deviation and extreme values of response quantities is demonstrated. It is found that the hydroelastic behaviour of very large floating structures is greatly affected when considering a directional wave spectrum, which provides a realistic description of the sea state. The developed framework can be applied to the study of very large floating structures with non-rectangular shapes as well as to investigate the behavior of very large floating structures with flexible connector systems. Future extensions may include the response to non-stationary sea states as well as the application of nonlinear random vibration analysis to account for the effect of second order wave forces.

\section{Acknowledgements}

This work was supported by the International Graduate School of Science and Engineering of the Technische Universität München. This support is gratefully acknowledged.

\section{References}

[1] Wang, CM, Watanabe, E, Utsunomiya, T. Very Large Floating Structures. Abingdon: Taylor \& Francis; 2008.

[2] Watanabe, E, Utsunomiya, T, Wang, CM. Hydroelastic analysis of pontoon-type VLFS: a literature survey. Engineering Structures 2004; 26(2):245-256.

[3] Newman, JN. Wave effects on deformable bodies. Applied Ocean Research 1994; 16(1): 47-59. 
[4] Hamamoto, T. Stochastic fluid-structure interaction of large circular floating islands during wind waves and seaquakes. Probabilistic Engineering Mechanics 1995; 10(4): 209-224.

[5] Chen, XJ, Jensen, JJ, Cui, WC, Tang, XF. Hydroelastic analysis of a very large floating plate with large deflections in stochastic seaway. Marine Structures 2004; 17(6):435454.

[6] Chen, XJ, Moan, T, Fu, SX, Cui, WC. Second-order hydroelastic analysis of a floating plate in multidirectional irregular waves. International Journal of Non-Linear Mechanics 2006; 41(10):1206-1218.

[7] Chen, XJ, Moan, T, Fu, SX. Extreme response of Very Large Floating Structure considering second-order hydroelastic effects in multidirectional irregular waves. Journal of Offshore Mechanics and Arctic Engineering-Transactions of the ASME 2010; 132(4): 041601.1-041601.11.

[8] Liew, KM, Wang, CM, Xiang, Y. Vibration of Mindlin Plates: Programming the pversion Ritz Method. Oxford: Elsevier; 1998.

[9] Sarpkaya, T, Isaacson, M. Mechanics of Wave Forces on Offshore Structures. New York: Van Nostrand Reinhold Co.; 1981.

[10] Faltinsen, OM. Sea Loads on Ships and Offshore Structures. Cambridge: Cambridge University Press; 1990.

[11] Eatock Taylor, R, Waite, JB. The dynamics of offshore structures evaluated by boundary integral techniques. International Journal for Numerical Methods in Engineering 1978; 13(1): 73-92.

[12] Linton, CM. Rapidly convergent representations for Green's functions for Laplace's equation. Proceedings of the Royal Society of London. Series A: Mathematical, Physical and Engineering Sciences 1999; 455: 1767-1797.

[13] Brebbia, CA, Telles, JCF, Wrobel, LC. Boundary Element Techniques: Theory and Applications in Engineering. Berlin: Springer-Verlag; 1984.

[14] Hinton, E, Huang, HC. A family of quadrilateral Mindlin plate elements with substitute shear strain fields. Computer \& Structures 1986; 23(3): 409-431.

[15] Mitsuyasu, H. On the growth of spectrum of wind generated waves (2) - spectral shapes of wind waves at final fetch. In: Proc 17th Japanese Conference on Coastal Engineering 1970; 1-7 (in Japanese). 
[16] Pierson, WJ, Tuttle, JJ, Wooley, JA. The theory of the refraction of a short-crested Gaussian sea surface with application to the northern New Jersey coast. In: Proc 3rd Conference on Coastal Engineering, Cambridge, MA, 1952; 86-108.

[17] Lutes, LD, Sarkani, S. Random Vibrations. Analysis of Structural and Mechanical Systems. Burlington, MA: Elsevier Butterworth-Heinemann; 2004

[18] Vanmarcke, EH. On the distribution of the first-passage time for normal stationary random processes. Journal of Applied Mechanics ASME 1975; 42(1): 215-220.

[19] Naess, A. The joint crossing frequency of stochastic processes and its application to wave theory. Applied Ocean Research 1985; 7(1): 35-50.

[20] Sim, IH, Choi, HS. An analysis of the hydroelastic behavior of large floating structures in oblique waves. In: Proc 2nd International Conference on Hydroelasticity in Marine Technology, Fukuoka, Japan, 1998; 195-199.

[21] Gao, RP, Tay, ZY, Wang, CM, Koh, CG. Hydroelastic response of very large floating structure with a flexible line connection. Ocean Engineering 2011; 38(17-18): 19571966.

[22] Ramesh, SS, Wang, CM, Reddy, JN, Ang, KK. Computation of stress resultants in plate bending problems using higher-order triangular elements. Engineering Structures 2008; 30(10): 2687-2706. 